\title{
Perspektiven für Hauptschulabsolventen
}

\section{Ergebnisse des Modellprojekts »Servicehelfer im Sozial- und Gesundheitswesen «}

\author{
Melanie Schuster
}

In einem Modellprojekt der RobertBosch-Stiftung werden Jugendliche mit Hauptschulabschluss aber obne Ausbildungsstelle für unterstützende Tätigkeiten in der Alten-und Behindertenhilfe und im Krankenhaus ausgebildet. Dabei geht es nicht um pflegerische Tätigkeiten, sondern um allgemeine Servicetätigkeiten.

In Deutschland verlassen jährlich über 75.000 Jugendliche die Schule ohne Abschluss, die Konsequenz heißt oft Sozialamt und Perspektivlosigkeit. Selbst ein Hauptschulabschluss bietet keine Garantie für einen Ausbildungsplatz.

Gerade Jugendliche mit weniger guten Abschlusszeugnissen haben es schwer, auf dem Ausbildungs- und Arbeitsmarkt Fuß zu fassen. Statt einer Ausbildung zieht ein Großteil der Hauptschulabsolventen Schleifen im Übergangssystem, das getrost als Maßnahmendschungel bezeichnet werden kann. So haben 40 Prozent der Hauptschulabsolventen auch eineinhalb Jahre nach dem Abschluss noch keinen Ausbildungsplatz und damit kaum Chancen auf dem Arbeitsmarkt. In einem Verdrängungswettbewerb um Ausbildungsplätze stehen Hauptschulabsolventen mit immer schlechteren Chancen da.

Auf der anderen Seite vermelden viele Betriebe im Sozial- und Gesundheitswesen einen großen Bedarf an Servicekräften. Laut Arbeitsmarktberichterstattung der Bundesagentur für Arbeit (Januar 2009) stieg die Nachfrage nach Fachpersonal im Sozial- und Gesundheitswesen weiter an. Dienstleistungen für alte, kran-

Melanie Schuster ist Koordinatorin des Projekts »Servicehelfer im Sozial- und Gesundheitswesen « der Robert Bosch Stiftung GmbH in Stuttgart.

E-Mail

melanie.schuster@bosch-stiftung.de ke und behinderte Menschen lassen sich nicht "outsourcen «, dieser Bedarf wird auch in Zukunft wachsen.

Der demografische Wandel macht deutlich, dass immer weniger junge Menschen für Dienstleistungen zur Verfügung stehen werden. Alle Jugendlichen werden gebraucht, auch wenn sie keine glänzenden Abschlusszeugnisse aufweisen können.

Ausgehend von diesen Bedarfslagen hat die Robert Bosch Stiftung das Modellprojekt "Servicehelfer im Sozial- und Gesundheitswesen « entwickelt, das Hauptschulabsolventen für unterstützende Tätigkeiten in der Alten- und Behindertenhilfe und im Krankenhaus qualifiziert und damit der Perspektivlosigkeit vieler Jugendlicher ein neues Konzept entgegen setzen will. Ziel ist, die Jugendlichen mit einem auskömmlichen Gehalt dauerhaft in den ersten Arbeitsmarkt des Sozialund Gesundheitswesens zu vermitteln.

Eckpfeiler des Modellprojekts sind eine klar umschriebene Zielgruppe (Hauptschulabsolventen, die aufgrund ihrer schulischen Leistungen oder aus anderen Gründen keine Lehrstelle finden), eine auf sie zugeschnittene Ausbildung und ein Curriculum, das diese Rahmenbedingungen berücksichtigt. Nach der einjährigen Qualifizierungsphase folgt ein Jahr Bewährung auf dem Arbeitsmarkt als fester Bestandteil des Modellprojekts.

Zentrales Element des Projekts ist ein Kooperationsverbund, der aus Trägervertretern der am Modell beteiligten Einrichtungen sowie einzelnen Experten aus Hauptschule, Bildung und Sozialministerium besteht. Der Verbund ist an der Weiterentwicklung des Modellkonzepts sowie an der Begleitung und Steuerung beteiligt. Zehn Träger der Alten- und Behindertenhilfe sowie der Krankenpflege, darunter das Wohlfahrtswerk für Baden-Württemberg, bieten in Einrichtungen im Großraum Stuttgart, Reutlingen und Augsburg Ausbildungsplätze an.
Die Jugendlichen im Modellprojekt lernen ein Jahr lang durch praktisches Tun in den jeweiligen Einrichtungen (70\%) und durch Theorieunterricht in der Schule $(30 \%)$. Der theoretische Unterricht findet in Blöcken statt, im Durchschnitt drücken die Servicehelfer stets zwei Wochen die Schulbank und sind anschließend vier bis sechs Wochen in der Praxis, um die in der Theorie erworbenen Fähigkeiten umzusetzen und zu vertiefen.

Das Curriculum wurde mit Blick auf die Zielgruppe und auf den Bedarf in der Praxis eigens entwickelt und unterliegt einem kontinuierlichen Revisionsprozess. In der Schule wird beispielsweise Hintergrundwissen zu alten, behinderten und kranken Menschen vermittelt; richtiger Umgang und kreative Beschäftigungsmöglichkeiten mit Bewohnern und $\mathrm{Pa}$ tienten werden ebenso erlernt wie das $\mathrm{Zu}$ bereiten von kleinen Mahlzeiten. Wichtige Regeln für Transportdienste, der Umgang mit Werkzeug und Geräten und Hygiene-Richtlinien stehen genauso auf dem Stundenplan wie ein Erste-HilfeKurs und grundlegende Kommunikationsregeln zur Stärkung der sozialen Kompetenz.

In den praktischen Einrichtungen werden die Jugendlichen in einem oder mehreren der folgenden Bereiche eingesetzt: - patienten- und bewohnernahe Tätigkeiten

- serviceorientierte und hauswirtschaftliche Tätigkeiten

- (haus-)technische und logistische Tätigkeiten

- administrative Tätigkeiten

Servicehelfer, die patienten- und bewohnernahe Tätigkeiten durchführen, begleiten diese beispielsweise zu Veranstaltungen, zu Arztbesuchen oder bei Einkäufen, lesen vor, gehen mit Patienten und Bewohnern spazieren oder helfen bei der Auswahl von Speisen.

$\mathrm{Zu}$ serviceorientierten und hauswirtschaftlichen Tätigkeiten gehören das Reinigen von Rollstühlen, Betten machen, 
das Zubereiten von kleineren Mahlzeiten oder Kaffee, Tische decken, Wäsche verteilen und die Mithilfe in der Cafeteria.

Servicehelfer, die (haus-) technische und logistische Tätigkeiten durchführen, bringen Material ins Labor, hängen Bilder auf, wechseln kaputte Glühbirnen aus, kümmern sich um Pflanzen und Außenanlagen oder trennen und entsorgen Abfall.

Unter administrative Tätigkeiten fallen beispielsweise das Anfertigen von Kopien oder das Sortieren von Akten und Karteikarten. gleitend und ergebnisorientiert untersucht werden. Die damit beauftragten Evaluatoren gehen beispielsweise der Frage nach, ob sich die Ausbildung und der Einsatz letzten Endes für Einrichtungsträger und auch aus Qualitätsgesichtspunkten heraus für ältere, kranke und behinderte Menschen rechnen.

Nach Auswahlgesprächen und kurzen Hospitationen in den Einrichtungen haben 22 junge Menschen am 1. November 2007 mit der Ausbildung begonnen. Die Jugendlichen hatten zum Auswahlzeitraum - längstens nach den Sommerfe-

\section{»Alle Jugendlichen werden gebraucht, auch wenn sie keine glänzenden Abschlusszeugnisse haben "}

Die Servicehelfer sollen das Pflegepersonal durch Tätigkeiten entlasten, die nicht originär zur Pflege gehören. Es geht also keinesfalls darum, Billigpflege anzubieten. Durch den verbesserten Kontakt zu Bewohnern und Patienten und die damit einhergehende Serviceverbesserung erwarten sich die Einrichtungen eine Qualitätsverbesserung für ihre Kunden.

Die Servicehelfer sind während ihrer Ausbildung nicht auf sich alleine gestellt: In den Einrichtungen werden sie von Praxisanleitern, die auf die spezifischen Anforderungen der Jugendlichen eingehen, unterstützt und angelernt. Hinzu kommt eine intensive pädagogische Betreuung der Servicehelfer seitens der Schule, die stark nachgefragt wird. Falls es bei einzelnen Servicehelfern größere Probleme gibt, steht eine übergeordnete pädagogische Fachkraft als Ansprechpartnerin und neutrale Instanz zur Verfügung. Falls nötig, wird an weiterführende Beratungsstellen vermittelt.

Das Modellprojekt, mit dem die Berufsschulpflicht abgegolten ist, sieht das erste Beschäftigungsjahr als zentralen Bestandteil des Modells. Anschließend erfolgt die staatliche Anerkennung der Ausbildung.

Besonders wichtig erscheint, dass die Voraussetzungen für einen Erfolg des Modellprojekts ausführlich prozessbe- rien - keinen Ausbildungsplatz gefunden und deshalb die Eingangskriterien erfüllt. Bei der Akquise der Jugendlichen hat sich vor allem die direkte Ansprache durch Lehrer als zielführend erwiesen.

60 Prozent der Servicehelfer des ersten Durchgangs haben das Jahr theoretische und praktische Ausbildung "geschafft « und schließlich die Prüfungen mit einem Notendurchschnitt von 2,4 bestanden. Als Lohn ihres Durchhaltevermögens folgte zum 1. November 2008 die mindestens einjährige Anstellung in den jeweiligen praktischen Einrichtungen.

In den zweiten Durchgang des Modellprojekts, der im Oktober 2008 begonnen hat, fließen erste Evaluierungsergebnisse ein. Auch im zweiten Durchgang wurden 22 Servicehelfer aufgenommen. Momen$\tan$ (Februar 2009) sind noch alle Jugendlichen engagiert dabei und nutzen ihre Chance, sich im Berufsleben zu etablieren.

Weitere Informationen: Internet http://www. bosch-stiftung.de/servicehelfer.

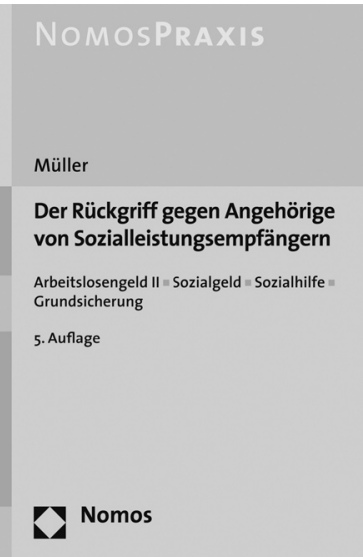

\section{Der Rückgriff gegen Angehörige von Sozial- leistungsempfängern} Arbeitslosengeld II, Sozialgeld, Sozialhilfe, Grundsicherung Von Prof. Dr. Christian Müller 5. Auflage 2008, $248 \mathrm{~S}$., brosch., 34,- €, ISBN 978-3-8329-2922-O

Die Frage, ob und unter welchen Voraussetzungen das Sozialamt erbrachte Geldleistungen von den (potentiell) unterhaltspflichtigen Angehörigen zurückfordern kann, hat in den letzten Jahren stetig an Bedeutung gewonnen. Die Neuauflage untersucht nicht nur die Rückgriffsmöglichkeiten beim Sozialhilfebezug, sondern auch die unterhaltsrechtlichen Auswirkungen im Rahmen des SGB II.

Im Einzelnen behandelt der Band

- die Grundzüge des Unterhaltsrechts nach der Reform,

- die Möglichkeiten und Grenzen der Inanspruchnahme naher Angehöriger beim Bezug von Sozialhilfe,

- den Regress beim Bezug von Arbeitslosengeld II und Sozialgeld nach SGB II,

- den Einfluss von Unterhaltsansprüchen bei der Gewährung von Leistungen der Grundsicherung im Alter und bei Erwerbsminderung nach SGB XII.

\section{Nomos \\ Bitte bestellen Sie im Buchhandel ode} versandkostenfrei unter $\downarrow$ www.nomos-shop.de 